\title{
Autophagy Promotes Survival of CHP-212 Neuroblastoma Cells Treated With Casiopeínas ${ }^{\circledR}$
}

\author{
ADRIANA VÁZQUEZ-AGUIRRE ${ }^{1}$, ANLLELY GRIZETT GUTIÉRREZ ${ }^{2}$, RAFAEL MORENO ESPARZA ${ }^{3}$, \\ ENRIQUE HERNÁNDEZ-LEMUS ${ }^{4,5}$, LENA RUIZ-AZUARA ${ }^{3}$ and CARMEN MEJÍA ${ }^{1}$ \\ ${ }^{1}$ Cell Biology Laboratory, Faculty of Natural Sciences, Autonomous University of Querétaro, Querétaro, Mexico; \\ ${ }^{2}$ Biomedical Research Center, Universidad Veracruzana, Jalapa, México; \\ ${ }^{3}$ Department of Inorganic and Nuclear Chemistry, Faculty of Chemistry, \\ National Autonomous University of Mexico, Mexico City, Mexico; \\ ${ }^{4}$ Department of Computational Genomics, National Institute of Genomic Medicine, Mexico City, Mexico; \\ ${ }^{5}$ Center of Complexity Sciences, National Autonomous University of Mexico, Mexico City, Mexico
}

\begin{abstract}
Background: Neuroblastoma is the main solid extracranial tumor of childhood. The amplification of $N$-myc oncogene (MYCN) and $1 p$ deletion are the main molecular alterations. These features are what make treatment impossible, especially in high-risk patients with metastases. Materials and Methods: Our study investigated the processes undergone by CHP-212 neuroblastoma cells, after being treated with Casiopeinas ${ }^{\circledR}$ (Cas) IIgly, IIIEa, and IIIia for 2, 10, and $24 \mathrm{~h}$. Results: At $2 \mathrm{~h}$, all the treatments Ied to apoptosis [defined by the presence of B-cell lymphoma 2 (BCL2), BCL2-associated X protein, cytochrome $c$, and caspase-3]. In addition, autophagy with specific molecules beclin-1 and microtubule-associated protein 1A/1B-light chain 3 (LC3)-II/LC3-I (ratio >1). Later at $10 \mathrm{~h}$, autophagyassociated proteins were observed, and at 24 h, only survival proteins nuclear factor kappa-light-chain-enhancer of activated B-cells $(N F-K B)$, and extracellular signal-regulated kinases (ERK)2/ERKI>1 were found. Another relevant finding was the presence of caspase-10 throughout the study, especially in cells treated with CasIIgly and CasIIIEa. Conclusion: These relationships indicate a possible mechanism of action of Casiopeinas on neuroblastoma.
\end{abstract}

Neuroblastoma (NB) is one of the deadliest solid extra-cranial tumors in childhood. Its heterogeneity is due to factors such as the age of the patient at diagnosis, tumor histology or genetic alterations such as amplification of the $\mathrm{N}$-myc

Correspondence to: Dr. Carmen Mejía, Laboratory of Cell Biology, Faculty of Natural Sciences, Autonomous University of Querétaro, Querétaro, C.P. 76230, Mexico. E-mail: carmen.mejia.iib@gmail.com

Key Words: Neuroblastoma cells CHP-212, Casiopeínas ${ }^{\circledR}$, autophagy, survival, caspase-10. oncogene $(M Y C N)$ and deletion of $1 \mathrm{p}(1)$. All these features mean that treatments of high-risk patients and metastasis are ineffective mainly due to apoptosis deregulation, a fact that has become a challenge in seeking new treatments for NB.

This is why we decided to investigate the therapeutic use of Casiopeínas ${ }^{\circledR}$ (Cas), a family of copper(II) coordination compounds (2). These molecules have been shown to produce antiproliferative, cytotoxic, genotoxic and antineoplastic effects in in vivo (3) and in vitro (4) models, even at concentrations lower than those used in cisplatin therapy. Our previous studies showed that cell death occurs by the mitochondrial apoptotic pathway, especially in CHP212 NB cells and SK-N-SH, at 2 and $4 \mathrm{~h}$, respectively $(5,6)$. The mechanism of action of Casiopeínas also involves generation of reactive oxygen species (ROS) and direct interaction with DNA (7). In light of our results, we set out to explore the behavior of these compounds over the course of a day. Another possible phenomenon involved after $4 \mathrm{~h}$ of treatment is autophagy. Until recently, autophagy was considered a type of non-apoptotic cell death (8); however, its role in cancer has been found to be more complex. Autophagy in many solid tumor types, including NB, may constitute a survival mechanism that confers resistance to chemotherapeutic agents in order to remove damaged organelles and recycle nutrients (9). In this study, we aimed to investigate the effect of Casiopeínas on apoptosis, autophagy and survival of NB cells.

\section{Materials and Methods}

Reagents. [Cu (4,7-dimethyl-1,10-phenanthroline) (glycinate) $\left(\mathrm{H}_{2} \mathrm{O}\right) \mathrm{NO}_{3}$ (Casiopeína IIgly, CasIIgly), [Cu (4,7-dimethyl-1,10phenanthroline) (acetylacetonate) $\left.\left(\mathrm{H}_{2} \mathrm{O}\right)\right]_{\mathrm{NO}_{3}}$ (CasIIIEa), and $[\mathrm{Cu}$ (4,40-dimethyl-2,20-bipyridine) (acetylacetonate) $\left(\mathrm{H}_{2} \mathrm{O}\right) \mathrm{NO}_{3}$ (CasIIIia) (Figure 1) were synthesized and characterized according to previously reported procedures (2). Cisplatin was obtained from 
A

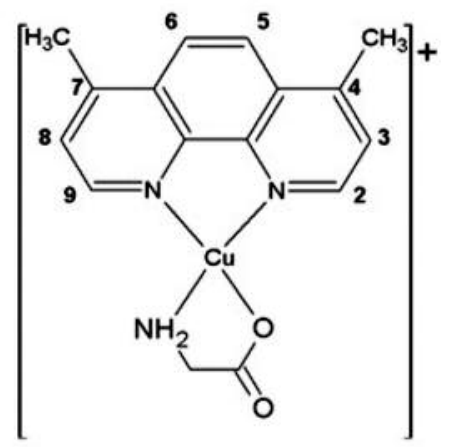

B

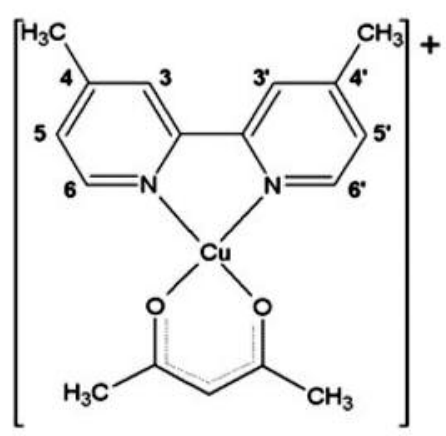

C

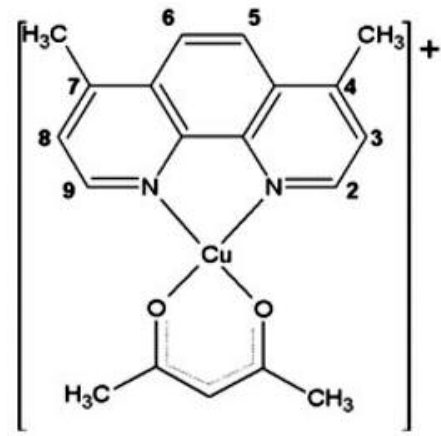

Figure 1. Casiopeinas used in this study. The structures of the three coordinated compounds with a metallic center of copper(II) used in this study are shown. A: Casiopeína IIgly [Cu(4,7-dimethyl-1,10-phenanthroline) (glycinate) ( $\left.\mathrm{H}_{2} \mathrm{O}\right) \mathrm{NOO}_{3}$; B: Casiopeína IIIia [Cu(4,40-dimethyl-2,20bipyridine) (acetylacetonate) $\left.\left(\mathrm{H}_{2} \mathrm{O}\right)\right] \mathrm{NO}_{3}$ and $\mathrm{C}$ : Casiopeína IIIEa [Cu (4,7-dimethyl-1,10-phenanthroline) (acetylacetonate) $\left.\left(\mathrm{H}_{2} \mathrm{O}\right)\right] \mathrm{NO}_{3}$.

Sigma-Aldrich (Sigma Chemical Co., St. Louis, MO, USA) and all the compounds were dissolved in sterile water.

Culture conditions and cell viability. CHP-212 cell line (directly sourced from the Rockville, MD, USA) was maintained under sterile conditions at $37^{\circ} \mathrm{C}$ and $5 \% \mathrm{CO}_{2}$ in Dulbecco's modified Eagle's medium, with $10 \%$ fetal bovine serum and F-12 medium (Sigma Chemical Co. St. Louis, MO, USA). Cells were treated for 2, 10 and $24 \mathrm{~h}$ with their mean $50 \%$ inhibitory concentrations $\left(\mathrm{IC}_{50}\right)$ of cisplatin, CasIIgly, CasIIIEa or CasIIIia as previously reported (5).

Western blot. Proteins of the cytoplasmic fraction were evaluated by western blot, prior subcellular fractionation (mitochondria/ cytosol fractionation; Calbiochem, Merck KGaA, Darmstadt, Germany). Briefly $5 \times 10^{6}$ treated cells were re-suspended in Cytosol Extraction Buffer Mix plus $1 \mathrm{M}$ dithiothreitol and cocktail of protease inhibitors (Calbiochem). Samples underwent polyacrylamide gel electrophoresis and then were transferred to nitrocellulose membrane. Subsequently, the membrane was incubated overnight at $4^{\circ} \mathrm{C}$ with primary antibodies against: apoptosis-associated proteins procaspases and caspases $(-3$, 7, -8, and -10), B-cell lymphoma 2 (BCL2), BCL2-associated X protein (BAX), and cytochrome $c$; autophagy-associated proteins beclin-1 [BECN1, a component of the phosphatidylinositol-3-kinase (PI3K) complex, which mediates vesicle-trafficking processes], microtubuleassociated protein 1A/1B-light chain 3 (LC3)-I and LC3-II,. as well as survival-associated proteins nuclear factor kappa-light-chain-enhancer of activated B-cells (NF-kB), extracellular signal-regulated kinase (ERK)1 and 2, and $\beta$-tubulin as loading control (all from Santa Cruz Biotechnology, Dallas, TX, USA). Bands were visualized on a film, using SuperSignal ${ }^{\circledR}$ West Dura Extended Substrate (Thermo Scientific, St. Louis, MO, USA) kit and measured with Image $\mathrm{J}^{\circledR}$ (NIH, WA, USA) version 1.50i. The results were expressed as relative units (RU), according to optical densitometry (OD $500 \mathrm{~nm}$ ).

Circos plot. In order to correlate all the variables under study, a Circos plot was created for the molecules of apoptosis, autophagy and survival in cells exposed to the different treatments.

Statistical analysis. Data are presented as averages \pm standard deviation (SD). All determinations were made in triplicate and the differences between groups were analyzed by ANOVA $(p<0.05)$.

\section{Results}

Apoptosis is a short-term process. The first finding was that procaspases $-3,-7$ and -8 and caspases -7 and -8 were not expressed (data not shown). In contrast, although all the cells expressed caspase-3, BCL2 and BAX, only those treated with cisplatin and CasIIgly had overexpression compared with the control (Figure 2A and B). Cytochrome $c$ was observed at two points of our analysis with some differences. After $2 \mathrm{~h}$, control cells did not express cytochrome $c$, while those treated with cisplatin or CasIIgly had low expression of cytochrome $c$ compared with cells treated with CasIIIEa or CasIIIia, which expressed up to 3and 5-fold more protein, respectively. At $10 \mathrm{~h}$, cells under all treatments expressed cytochrome $c$ although less than at $2 \mathrm{~h}$ (Figure 2C).

Autophagy is a longer process. This process was analyzed through the expression of BECN1, the main protein involved forming autophagosomes, and LC3-I, and LC3-II. An important parameter was the concomitant presence of BECN1 and BCL-2, since the latter can repress the expression of BECN1 and, therefore, autophagy. In our study, both proteins were expressed only at $2 \mathrm{~h}$ in cells treated or not. Interestingly, the control cells showed a very similar expression of BECN1 and BCL-2, while cells treated with Casiopeínas overexpressed BECN1, and those treated with cisplatin expressed BCL2 mainly (Figure 3A). When the expression of BECN1 was analyzed at $10 \mathrm{~h}$, we found it overexpressed by up to two-fold in cells treated with CasIIIEa and cisplatin, compared with the control. However, in cells treated with CasIIgly and CasIIIia, the expression of BECN1 was similar to that of the control cells (Figure 3C).

LC3-I and -II proteins are necessary for the elongation and fusion of autophagosome. Because the predominant 

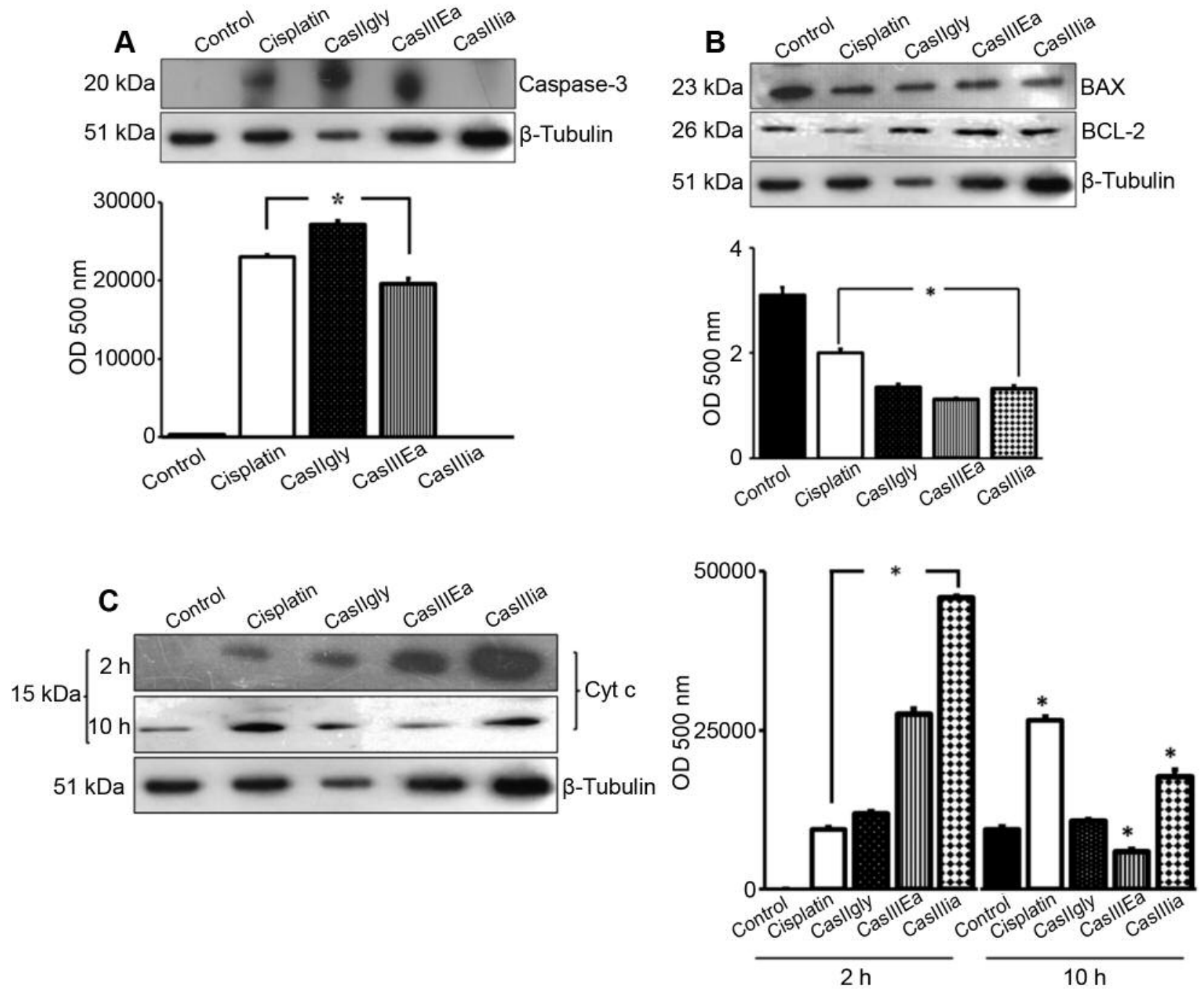

Figure 2. Apoptotic molecules appeared early in treatment. Determinations were performed for cell cultures treated with the half-maximal inhibitory concentration $\left(I C_{50}\right)$ of the three different Casiopeinas (Cas) or cisplatin, in addition to the control group that did not receive treatment. All values were obtained by normalizing versus $\beta$-tubulin $(15 \mathrm{kDa})$ as loading control. A: Cleaved caspase-3 was observed in all cases only at 2 h. Compared with the control group, the different treatments produced a greater amount of cleaved caspase-3, especially with CasIIgly. B: The expression of B-cell lymphoma 2 (BCL2)-associated X protein (BAX) was higher than that of the antiapoptotic protein BCL2, and the BAX/BCL2 ratio is shown in the lower panel. C: Finally, cytochrome $c(C y t c)$ was found during two stages of the study. At $2 \mathrm{~h}$ its expression was notable mainly for cells treated with CasIIIE a and CasIIIia; their expression decreased at $10 \mathrm{~h}$ under all conditions. All values are representative of three independent experiments. ${ }^{*} p<0.05$.

expression of LC3-II is the maturation marker of these vesicles, we report our findings as LC3-II/LC3-I RU. Our first finding was these proteins were present at both 2 and $10 \mathrm{~h}$ but not later, and their expression differed according to the treatment. Thus, at $2 \mathrm{~h}$, control cells presented a ratio of 3 compared to treated cells, which showed the order: CasIIIia > CasIIgly > CasIIIEa > cisplatin (relative LC3II/LC3-I ratios of $2,1.23,1.21$ and 0.74 , respectively) (Figure 3B). Another important finding was LC3-II was absent from control cells at $10 \mathrm{~h}$, whereas treated cells showed a higher relative concentration of LC3-II than LC3I, with mean ratios of 1.7 for CasIIIia $>1.4$ for CasIIIEa $>1.2$ for cisplatin $>0.9$ for CasIIgly (Figure 3D).

Cell survival is the ultimate response to treatment. After $24 \mathrm{~h}$ of treatment, only expression of the cell survival molecules NF-kB, ERK1 and ERK2 were found. NF-kB activates genes that regulate cell survival and proliferation. We observed that the expression of this protein increased by up to 10 times in cells treated with cisplatin, CasIIgly and the control compared 

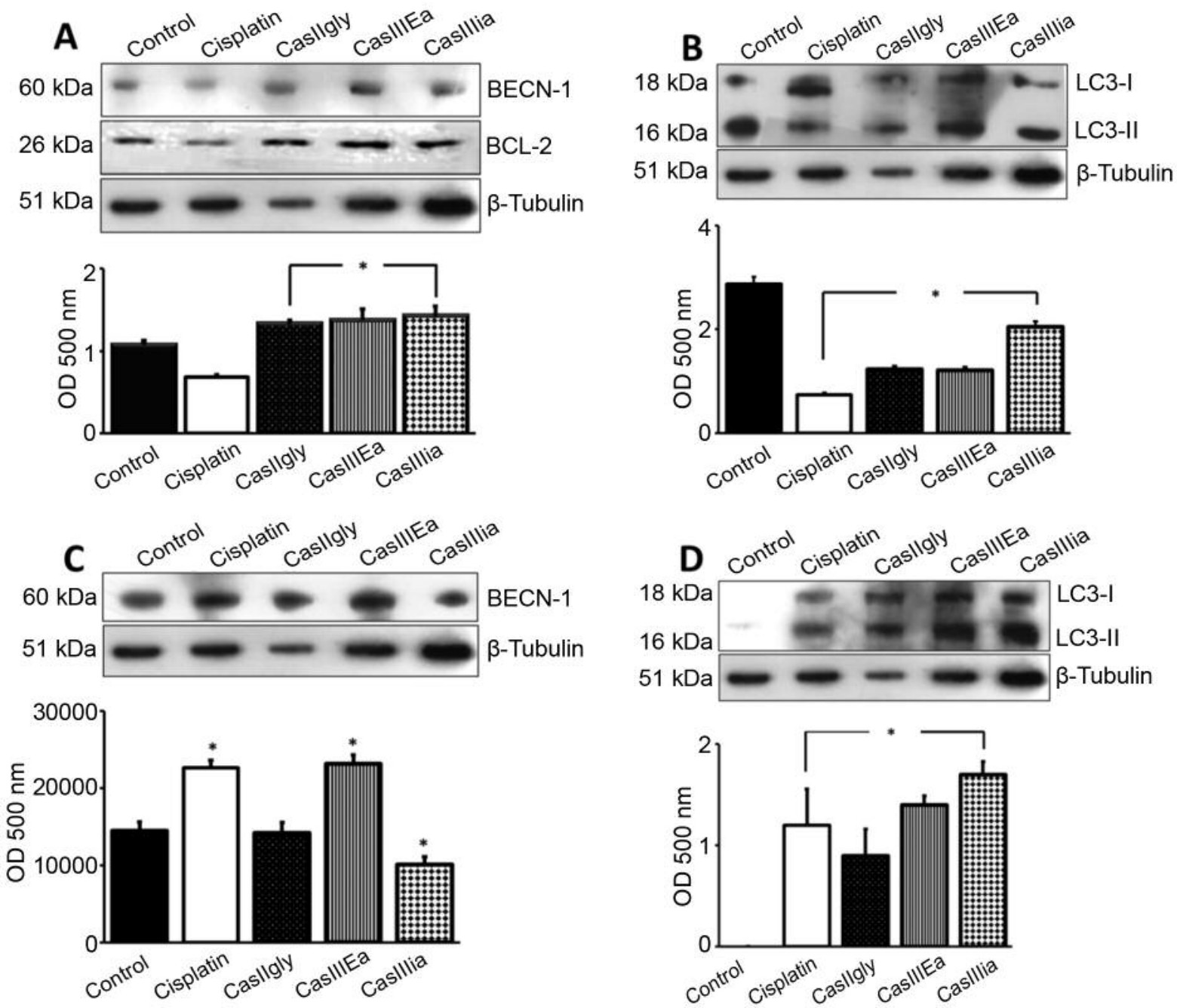

Figure 3. Autophagic protein determination after treatment with half-inhibitory concentration $\left(I_{50}\right)$ of all Casiopeinas (Cas) or cisplatin. At 2 h, an increment of the B-cell lymphoma 2 (BCL2)/beclin-1 (BECN1) ratio was found in all cells treated with Cas (A), as well as the beginning of the autophagic flow represented by the ratio of microtubule-associated protein 1A/1B-light chain 3 (LC3)-II/LC3-I (B). At 10 h BECN1 protein only decreased in neuroblastoma cells treated with CasIIIia $(C)$; and the maturation of the autophagic vesicles represented by the LC3-II/LC3-I ratio with an increase of LC3-II mainly. *Statistically significant at $p<0.05$ compared to control cells without treatment (D). Western blot quantification was performed by optical densitometry (O.D.). The determinations were carried out by normalizing the values with the loading control $\beta$-tubulin. All values are representative of three independent experiments. $* p<0.05$.

to those that received CasIIIEa or CasIIIIia (Figure 4A). On the other hand, the two isoforms of ERK are crucial components in the regulation of normal and malignant proliferation, although the difference in their concentrations may or may not promote survival. Therefore, even when the loss of ERK1 significantly facilitates proliferation, the inhibition of ERK2 in turn drastically reduces cell growth. Here we also report the ERK2/ERK1 ratio. In our study, we found a tendency towards survival promoted by an increased expression of ERK2 with respect to ERK1, especially in control cells (1.22 RU) and in those treated with CasIIIia (1.14 RU) or cisplatin (1.03 RU), whereas the cells that received CasIIgly and CasIIEa showed a slight increase in the expression of ERK1 (0.98 and 0.85 RU, respectively) (Figure 4B).

Expression of caspase-10 is sustained throughout $24 \mathrm{~h}$. Both procaspase-10 and its active form were expressed throughout the study, although with certain peculiarities. Thus at $2 \mathrm{~h}$, the expression of procaspase-10 was only found in control cells, while treatment with CasIIIia inhibited expression of caspase-10. After $10 \mathrm{~h}$, caspase- 10 was overexpressed in all cases by twofold than at $2 \mathrm{~h}$. 

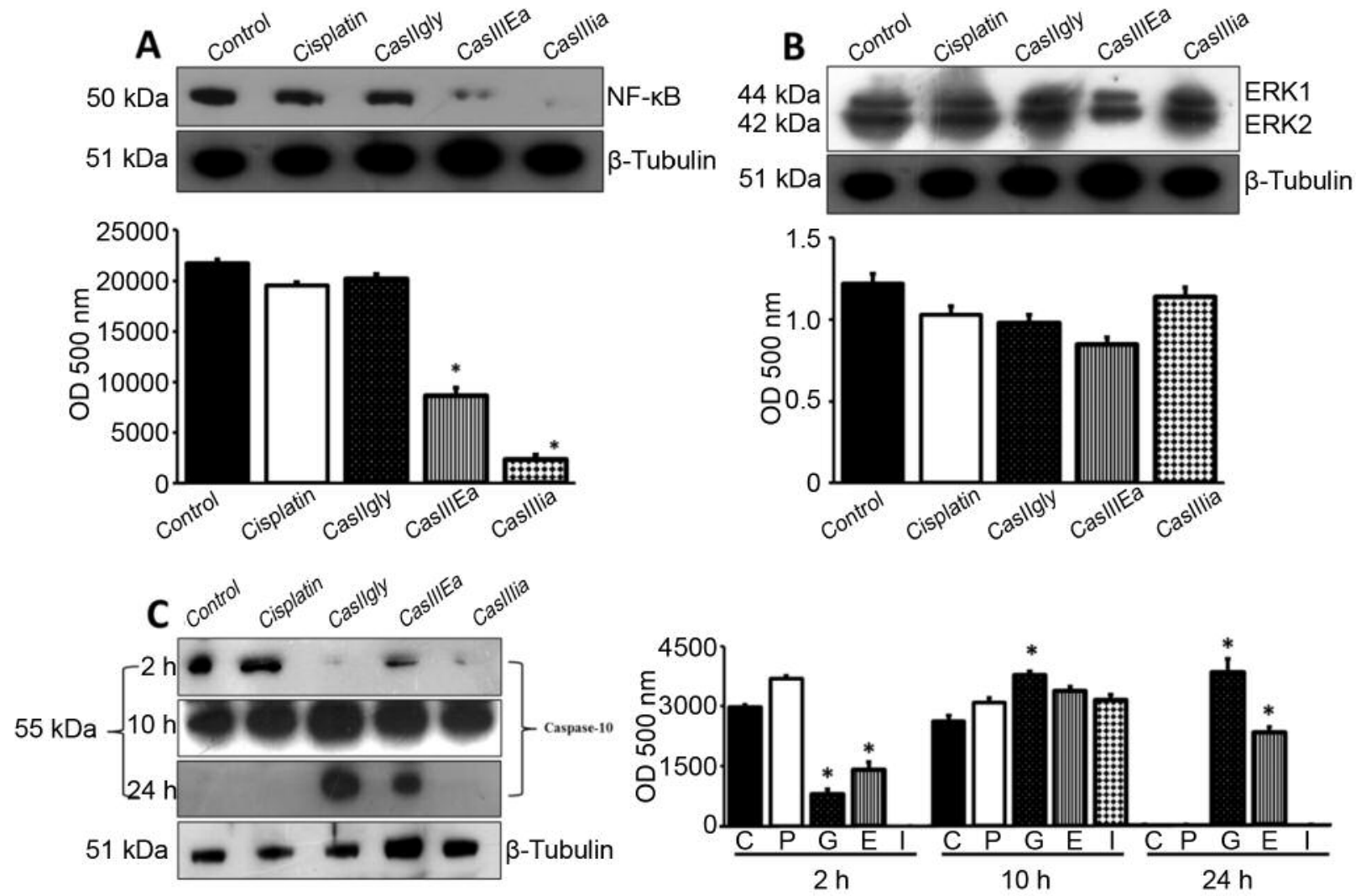

Figure 4. Survival proteins and caspase-10 post-treatment. A: Nuclear factor kappa-light-chain-enhancer of activated B cells (NF-kB) was found in most condition except for Casiopeinas (Cas) IIIEa and IIIia. B: Conversely, the extracellular signal-regulated kinase (ERK)2/ERK1 ratio was found to be higher under treatment with these Casiopeinas. C: Finally, caspase-10 was observed throughout the study with some differences. Thus, at $2 \mathrm{~h}$ caspase-10 was slightly expressed in treatments with CasIIgy and IIIEa, but was absent using Cas IIIia; meanwhile at 10 h, this caspase was overexpressed in all conditions. At 24 h, caspase-10 was observed only in treatments with CasIIgly and IIIEa and at a high proportion. C: Control; P: cisplatin; G: Casiopeína IIgly; E: Casiopeína IIIEa; I: Casiopeína IIIia. All determinations were performed by western blotting, and quantifications were carried out by normalizing the values with the loading control $\beta$-tubulin. Quantifications were measured by optical densitometry (O.D.). All values are representative of three independent experiments. ${ }^{*} p<0.05$.

Finally, caspase-10 was found at $24 \mathrm{~h}$ only in cells treated with CasIIgly and CasIIIEa, in a similar proportion to that observed at $10 \mathrm{~h}$ (Figure 4C).

By using Circos plot visualizations, we show all the relationships of our study. We noted the progression from apoptosis to autophagy and then to the survival states, marked by the areas in Figure 5 that go from red to dark blue, in a clockwise direction starting from the top, and which were determined by the of the associated proteins. The slats show the correlations between these states and the treatments (dark yellow to light blue areas) in the anticlockwise direction. The RUs of the proteins are shown on the scale of the inner circle, and the percentage correlations on the scale of the outer circle (Figure 5). Finally, we propose a mechanism of action for cells treated with CasIIgly and CasIIIEa at 2, 10 and $24 \mathrm{~h}$ (Figure 6).

\section{Discussion}

Regarding autophagy, several authors concluded that around $10 \mathrm{~h}$ after the start of the process is still visible $(10,11)$, which is why we defined this time as an intermediate point of our analysis. An important finding was that both apoptosis and autophagy were concomitant processes at $2 \mathrm{~h}$. It is likely that Casiopeínas play a double role in CHP- 212 cells that on the one hand allows them to enter apoptosis, but at the same time a significant proportion of cells are undergoing autophagy. This is understandable since both routes share molecules of the BCL2 family, such as BCL2, BAX and BECN1. For example, in MDA-MB-231 breast cancer cells, it has been reported that after Taxol chemotherapy, autophagy was activated before apoptosis, but only one of these pathways was dominant (12). In our study, apoptosis was the 


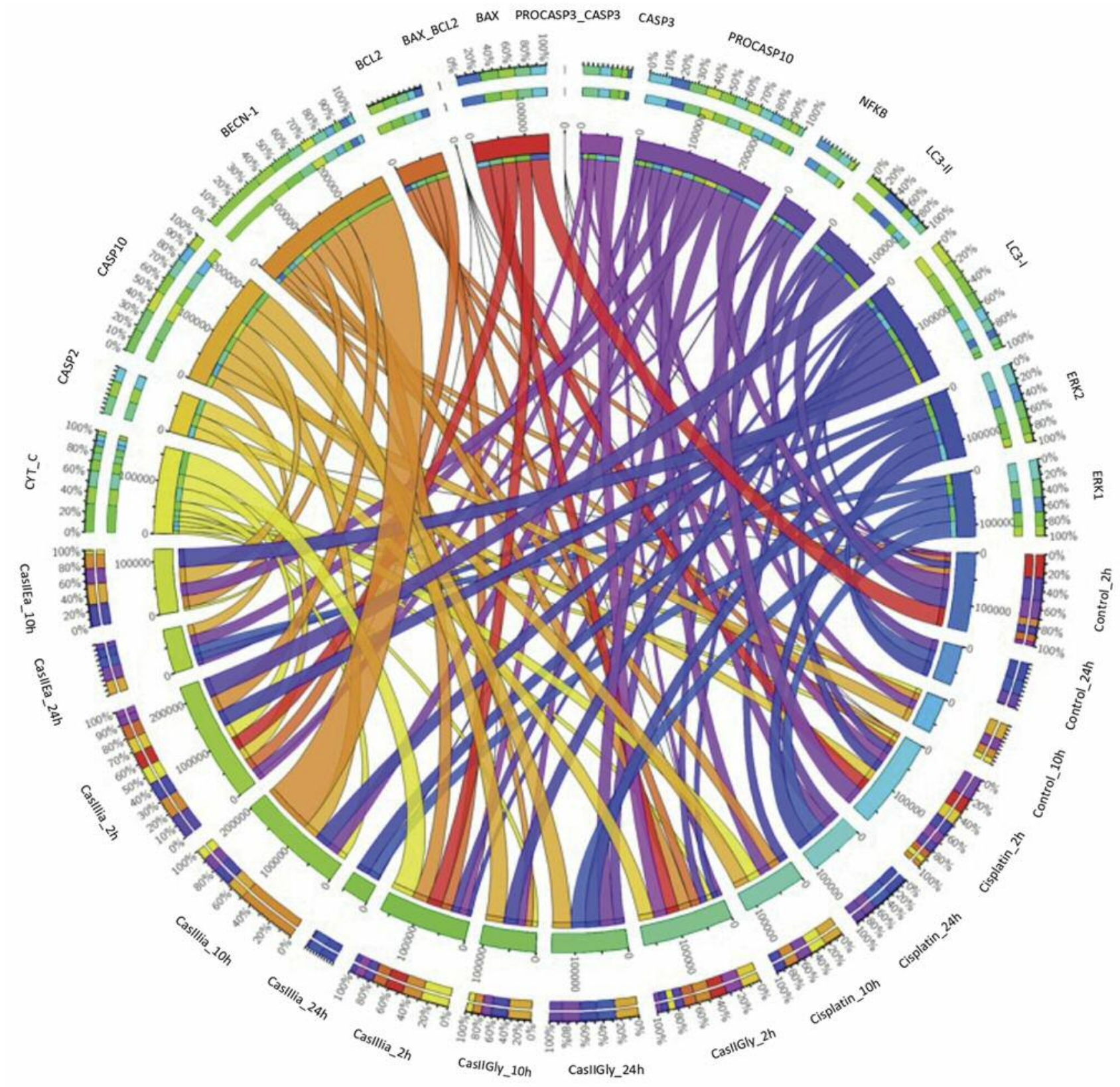

Figure 5. Circos plot of the relationships found with all the variables under study showing progression from apoptosis to autophagy and then to survival. Variables analyzed were: Casiopeina (Cas), caspases (CASP), pro-caspase (PROCASP), B-cell lymphoma 2 (BCL2)-associated X protein $(B A X)$, cytochrome c $(C y t c)$, beclin-1 (BECN1; component of the phosphatidylinositol-3-kinase (PI3K) complex, which mediates vesicle-trafficking processes), microtubule-associated protein 1A/1B-light chain 3 (LC3)-I and-II, nuclear factor kappa-light-chain-enhancer of activated B-cells (NF$\kappa B)$, and extracellular signal-regulated kinase (ERK) 1 and ERK2. The areas ranging from red to dark blue, in a clockwise direction starting from the top, represent means of the expression of associated proteins as relative units (RU). The slats show the correlations between these states and the treatments (dark yellow to light blue areas) in an anticlockwise direction. The RUs of the proteins are shown on the scale of the inner circle, and the percentage correlations on the scale of the outer circle.

main pathway activated by the Casiopeínas. It is known that proteins of the BCL2 family, which kidnap BECN1, posttranscriptionally regulate the autophagy. On the other hand, the release of BCN1 may be the result of the expression of the transcriptional repressor BCL2-associated transcription factor 1 (BCLAF1). So, it is plausible that Casiopeínas are involved in this mechanism. This proapoptotic protein (BH3only) can, in turn, compete for the binding of BCN1 to 


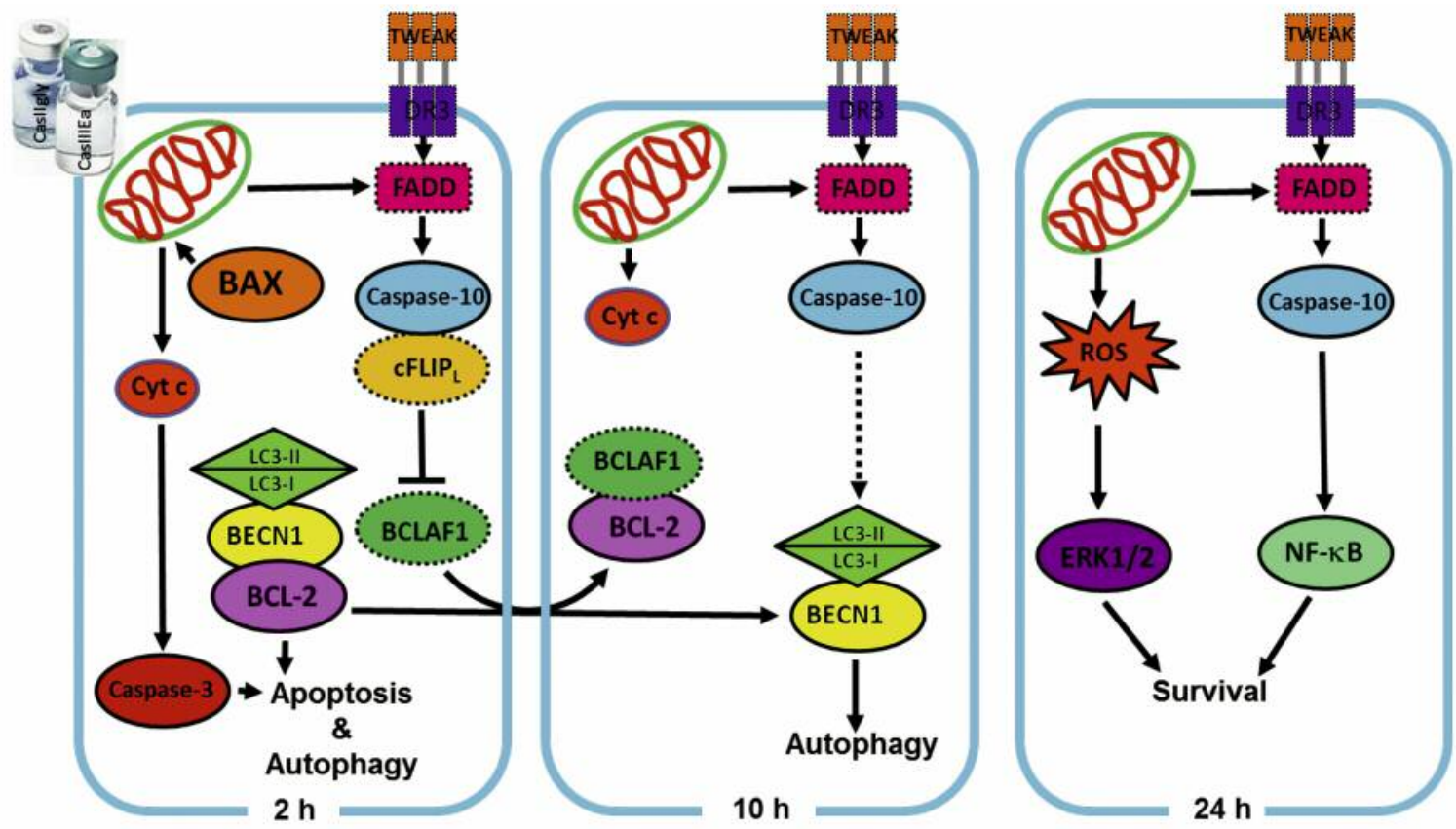

Figure 6. Proposed mechanism of action of Casiopeina (Cas)IIgly and (Cas) IIIEa in CHP-212 cells. After 2 h of treatment with Casiopeinas, apoptosis was observed as shown by the expression of caspase-3 and the co-expression of B-cell lymphoma 2 (BCL2)-associated X protein (BAX), cytochrome $c(C y t c)$, beclin-1 (BECN1; component of the phosphatidylinositol-3-kinase (PI3K) complex which mediates vesicle-trafficking processes), and B-cell lymphoma 2 (BCL2). The Fas-associated protein with death domain (FADD) adapter, which may be activated by the complex TNF-related weak inducer of apoptosis (TWEAK)/death receptor 3 (DR3), or by the same mitochondria, may activate caspase-10 upstream but in both cases it does not intervene in apoptosis. Subsequently, at $10 \mathrm{~h}$, the cells enter autophagy and no longer express BCL2 or BCL2-associated X protein $(B A X)$, but cytochrome $c$ and caspase-10 again, which may activate autophagy. Finally, the cells only express the survival proteins nuclear factor kappa-light-chain-enhancer of activated B cells $(\mathrm{NF}-\mathrm{k} B)$, and (extracellular signal-regulated kinase (ERK)1/2, which are activated by reactive oxygen species (ROS) or by the action of caspase-10, all this after $24 \mathrm{~h}$ of treatment. The molecules that were not determined in this study are identified with dotted figures.

BCL2; however, this competition is inhibited by caspase-10, which dimerizes with cellular Fas-associated protein with death domain (FADD)-like interleukin-1 $\beta$-converting enzyme-inhibitory protein (long variant) (cFLIPL) cleaving BCLAF1 protein (13). This seems to be the most plausible explanation for the events observed during the first phase of our study. In addition to BECN1, from the first moment we found slightly increased expression of LC3-II, indicative of the maturation of autophagosomal vesicles, and which is considered the main biochemical parameter of autophagy. However, the conversion of LC3-I to LC3-II does not necessarily result in complete autophagy or a pathway for degradation of damaged cellular material.

With respect to cytochrome $c$, it is well known that only up to 15 to $20 \%$ is found in the intermembrane space of mitochondria, a highly compartmentalized organelle. In our study, we found twice more cytochrome $c$ at 2 than at $10 \mathrm{~h}$, and although several studies suggest that cytochrome $c$ output is rapid and complete, it is known that only a low concentration of this protein is needed in the cytosol for apoptosis to take place (14). The explanation that cytochrome $c$ was present in two phases may be due to the fact that the exit of cytochrome $c$ from the mitochondria occurs in two parts, both induced by proapoptotic members of the BCL2 family. In the first, cytochrome $c$ contained in the intermembrane space exits the cytosol through the outer mitochondrial membrane. During the second phase, there is a release and redistribution of cytochrome $c$ that until then was sequestered in other compartments, such as the mitochondrial ridges of the inner membrane. This phenomenon leads to structural remodeling of the mitochondrial inner membrane and changes in the binding of cytochrome $c$ with membrane lipids such as cardiolipin. This mitochondrial remodeling could have been caused by truncated $\mathrm{BH} 3$ interacting-domain death agonist (tBID), and it seems to be independent of both BAX and BAK, which would cause the mobilization and release of the remaining cytochrome $c$ from inside the mitochondrial crests to the cytosol (14).

Another important finding was the presence of caspase-10 throughout the study. To date, there are few reports in which caspase-10 is described as an independent protein of apoptosis. 
It has been reported that in myeloma cells, this caspase is responsible for the cellular response preventing cell death (15). Our results showed that at the last point of the study, the expression of caspase-10 was related to the survival process due to the presence of NF-KB and ERK1/2 proteins. In myeloma cells, it has been observed that the factor interferon regulatory factor 4 induces the transcription of caspase-10 and its associated protein cFLIPL, generating a protease that does not induce apoptosis, but blocks a pathway dependent on autophagy. While myeloma cells require a basal level of autophagy to survive, caspase-10 can modulate this response to prevent cell death (15), so compounds that break this subtle balance are candidates with therapeutic potential in NB. In our study, we do not know what the adapter molecule that regulates caspase-10 is. One proposal comes from our whole-genome experiments, where we analyzed gene-expression microarrays of HeLa and NB cells treated with CasIIgly, and showed tumor necrosis factor (TNF)-related weak inducer of apoptosis (TWEAK) ligand and the death receptor DR3 together with the adapter molecule FADD as being responsible for the recruitment of caspase-8 (16). Caspase-10 has been considered a homolog of caspase-8, because both genes are located on chromosome 2q33-34 suggesting a tandem duplication event (17). It was recently shown that caspase-10 may also be involved in the mitochondrial pathway induced by cytotoxic drugs, activating downstream the mitochondria in a FADDdependent manner but through a mechanism independent of death receptor, where caspases 8 and 10 would be involved in the feedback of the amplification of the intrinsic pathway (18).

Finally, in CHP-212 cells, Cas treatment promoted the expression of NF-KB and ERK 1/2 proteins, whose activation favors cell survival. The presence of these survival molecules may be influenced by the expression of caspase-10 on the one hand, but also by the generation of ROS. It is known that caspases 8 and 10 have functions other than their proapoptotic role. Thus, it has observed that when both proteins are overexpressed, they can activate the NF-kB pathway. In the canonical pathway, it is believed that ROS activates NF-kBinducing kinase, the upstream kinase of NF- $\mathrm{KB}$, by inhibiting phosphatases and oxidizing cysteine residues. The NF-kB pathway may also influence ROS levels by increasing the expression of antioxidant proteins such as superoxide dismutase (SOD)-Cu-Zn, SOD-Mn, and glutathione peroxidase (19).

ERK1 and -2 belong to the family of mitogen-activated serine/threonine kinases, which mediate intracellular signal transduction in response to different physiological stimuli and stress conditions. However, ERK can play a double role in apoptosis. Thus, during apoptosis, caspase- 3 protects and inactivates RAF proto-oncogene serine/threonine-protein kinase, which leads to inhibition of the survival pathway. In contrast, the ERK pathway can act as an inhibitor of various apoptotic stimuli such as response to some chemotherapeutic agents (20), which our findings are consistent with.
In conclusion, in this work, we found that NB cell death induced by treatment with Casiopeínas is a very early process that allows the cell to carry out a rescue maneuver such as autophagy, to finally cleanse its system and be able to proliferate again. Such a decision is one that in the end can make the difference between the success or not of a chemotherapeutic agent.

\section{Conflicts of Interest}

The Authors have declared that no competing interests exist in regard to this study.

\section{Authors' Contributions}

EHL and CM made substantial contributions to the conception and design of the present study; LRA provided the Casiopeínas ${ }^{\circledR}$ in study; AVA, and AGG performed the experiments for data acquisition; RME, EHL and LRA performed the statistical analysis; AVA, AGG, EHL, and CM interpreted the experimental results; EHL, LRA, and CM wrote the article. The final version of the article was revised and approved by all Authors.

\section{Acknowledgements}

The Authors acknowledge to MsC Adrián Espinoza Guillén for the synthesis of Casiopeínas ${ }^{\circledR}$. This work was supported by FOFI-UAQ (87FCN2015), Red Temática Farmoquímicos CONACYT No. 294727 and, PAPIIT-UNAM IN218013.

\section{References}

1 Depuydt P, Boeva V, Hocking TD, Cannoodt R, Ambros IM, Ambros PF, Asgharzadeh S, Attiyeh EF, Combaret V, Defferrari R, Fischer M, Hero B, Hogarty MD, Irwin MS, Koster J, Kreissman S, Ladenstein R, Lapouble E, Laureys G, London WB, Mazzocco K, Nakagawara A, Noguera R, Ohira M, Park JR, Pötschger U, Theissen J, Tonini GP, Valteau-Couanet D, Varesio L, Versteeg R, Speleman F, Maris JM, Schleiermacher G and De Preter K: genomic amplifications and distal 6q loss: Novel markers for poor survival in high-risk neuroblastoma patients. J Natl Cancer Inst 110(10): 10841093, 2018. PMID: 29514301. DOI: 10.1093/jnci/djy022

2 Ruiz-Azuara L: Copper amino aminoacidate diimine nitrate compounds and their methyl derivatives and a process for preparing them. Application No. 07/628,628; US Patent 5,576,326, 1996.

3 Carvallo-Chaigneau F, Gómez-Ruiz C, Rodríguez-Aguilera E, Macías-Rosales L, Cortés-Barberena E, Cedillo-Peláez C, Gracia-Mora I, Ruiz-Azuara L, Madrid-Marina V and Constantino-Casas F: Casiopeina III-ia induces apoptosis in HCT-15 cells in vitro through caspase-dependent mechanisms and has antitumor effect in vivo. Biometals 21: 17-28, 2008. PMID: 17390215. DOI: 10.1007/s10534-007-9089-4

4 Trejo-Solís C, Palencia G, Zúñiga S, Rodríguez-Ropón A, Osorio-Rico L, Sánchez L, Gracia-Mora I, Márquez-Rosado L, Sánchez A, Moreno-García ME, Cruz A, Bravo-Gómez ME, Ruiz- Ramírez L, Rodríguez-Enríquez S and Sotelo J: Cas IIgly induces apoptosis in glioma C6 cells in vitro and in vivo through caspase-dependent and caspase-independent mechanisms. Neoplasia 7: 563-574, 2005. PMID: 16036107. 
5 Gutiérrez AG, Vázquez-Aguirre A, García-Ramos JC, FloresAlamo M, Hernández-Lemus E, Ruiz-Azuara L and Mejía C: Copper(II) mixed chelate compounds induce apoptosis through reactive oxygen species in neuroblastoma cell line CHP-212. J Inorg Biochem 126: 17-25, 2013. PMID: 23727332. DOI: 10.1016/j.jinorgbio.2013.05.001

6 García-Ramos JC, Gutiérrez AG, Vázquez-Aguirre A, ToledanoMagaña Y, Alonso-Sáenz AL, Gómez-Vidales V, Flores-Alamo M, Mejía C and Ruiz-Azuara L: The mitochondrial apoptotic pathway is induced by $\mathrm{Cu}(\mathrm{II})$ antineoplastic compounds (Casiopeínas ${ }^{\circledR}$ ) in SK-N-SH neuroblastoma cells after short exposure times. Biometals 30: 43-58, 2017. PMID: 27988860. DOI: $10.1007 / \mathrm{s} 10534-016-9983-8$

7 Kachadourian R, Brechbuhl HM, Ruiz-Azuara L, Gracia-Mora I and Day BJ: Casiopeína IIgly-induced oxidative stress and mitochondrial dysfunction in human lung cancer A549 and H157 cells. Toxicology 268: 176-83, 2010. DOI: 10.1016/j.tox.2009. 12.010

8 Galluzzi L, Kepp O, Trojel-Hansen C and Kroemer G: Nonapoptotic functions of apoptosis-regulatory proteins. EMBO Rep 13: 322-330, 2012. DOI: 10.1038/embor.2012.19

9 Rodríguez-Hernández MA, González R, de la Rosa ÁJ, Gallego P, Ordóñez R, Navarro-Villarán E, Contreras L, RodríguezArribas M, González-Gallego J, Álamo-Martínez JM, MarínGómez LM, Del Campo JA, Quiles JL, Fuentes JM, de la Cruz J, Mauriz JL, Padillo FJ and Muntané J: Molecular characterization of autophagic and apoptotic signaling induced by sorafenib in liver cancer cells. J Cell Physiol 234: 692-708, 2018. PMID: 30132846. DOI: 10.1002/jcp.26855

10 Li JY, Pan SS, Wang JY and Lu J: Changes in autophagy levels in rat myocardium during exercise preconditioning-initiated cardioprotective effects. Int Heart J 60: 419-428, 2019. PMID: 3074554. DOI: $10.1536 /$ ihj.18-310

11 Zhang P, Yang L, He H and Deng Y: Differential variations of autophagy and apoptosis in permanent focal cerebral ischaemia rat model. Brain Inj 31: 1151-1158, 2017. PMID: 28506087. DOI: $10.1080 / 02699052.2017 .1298005$

12 Notte A, Rebucci M, Fransolet M, Roegiers E, Genin M, Tellier C, Watillon K, Fattaccioli A, Arnould T and Michiels C: Taxolinduced unfolded protein response activation in breast cancer cells exposed to hypoxia: ATF4 activation regulates autophagy and inhibits apoptosis. Int J Biochem Cell Biol 62: 1-14, 2015. PMID: 25724736. DOI: 10.1016/j.biocel.2015.02.010

13 Maiuri MC, Zalckvar E, Kimchi A and Kroemer G: Self-eating and self-killing: Crosstalk between autophagy and apoptosis. Nat Rev Mol Cell Biol 8: 741-752, 2007. PMID: 17717517. DOI: $10.1038 / \mathrm{nrm} 2239$
14 Scorrano L, Ashiya M, Buttle K, Weiler S, Oakes SA, Mannella $\mathrm{CA}$ and Korsmeyer SJ: A distinct pathway remodels mitochondrial cristae and mobilizes cytochrome $c$ during apoptosis. Dev Cell 2: 55-67, 2002. PMID: 11782314.

15 Lamy L, Ngo VN, Emre NC, Shaffer AL, Yang Y, Tian E, Nair V, Kruhlak MJ, Zingone A, Landgren O and Staudt LM: Control of autophagic cell death by caspase-10 in multiple myeloma. Cancer Cell 23: 435-449, 2013. DOI: 10.1016/j.ccr.2013.02.017

16 Valencia-Cruz AI, Uribe-Figueroa LI, Galindo-Murillo R, BacaLópez K, Gutiérrez AG, Vázquez-Aguirre A, Ruiz-Azuara L, Hernández-Lemus E and Mejía C: Whole-genome gene expression analysis reveals casiopeína-induced apoptosis pathways. PLoS One 8: e54664, 2013. DOI: 10.1371/journal. pone. 0054664

17 Horn S, Hughes MA, Schilling R, Sticht C, Tenev T, Ploesser M, Meier P, Sprick MR, MacFarlane M and Leverkus M: Caspase-10 negatively regulates caspase-8-mediated cell death, switching the response to CD95L in favor of NF-kB activation and cell survival. Cell Rep 19: 785-797, 2017. PMID: 28445729. DOI: $10.1016 /$ j.celrep.2017.04.010

$18 \mathrm{Hu}$ TB, Chen HS, Cao MQ, Guo FD, Cheng XY, Han ZB and Li MQ: MicroRNA-421 inhibits caspase-10 expression and promotes breast cancer progression. Neoplasma 65: 49-54, 2018. PMID: 29322788. DOI: 10.4149/neo_2018_170306N159.

19 Salehi B, Martorell M, Arbiser JL, Sureda A, Martins N, Maurya PK, Sharifi-Rad M, Kumar P and Sharifi-Rad J: Antioxidants: Positive or negative actors? Biomolecules 8: pii: E124, 2018. DOI: $10.3390 /$ biom8040124

20 Abrams SL, Ruvolo PP, Ruvolo VR, Ligresti G, Martelli AM, Cocco L, Ratti S, Tafuri A, Steelman LS, Candido S, Libra M and McCubrey JA: Targeting signaling and apoptotic pathways involved in chemotherapeutic drug-resistance of hematopoietic cells. Oncotarget 8: 76525-76557, 2017. DOI: 10.18632/ oncotarget 20408
Received April 9, 2019

Revised May 22, 2019

Accepted May 28, 2019 